\title{
Stage IVB Mycosis Fungoides and Sezary Syndrome AJCC v8
}

National Cancer Institute

\section{Source}

National Cancer Institute. Stage IVB Mycosis Fungoides and Sezary Syndrome A/CC v8.

NCl Thesaurus. Code C141359.

Stage IVB includes: T1-4, N0-3, M1, B0-2. T1: Limited patches, papules, and/or plaques covering less than $10 \%$ of the skin surface. T2: Patches, papules, or plaques covering $10 \%$ or more of the skin surface. T3: One or more tumors (equal or greater than $1 \mathrm{~cm}$ in diameter). T4: Confluence of erythema covering $80 \%$ or more of body surface area. N0: No clinically abnormal peripheral lymph nodes; biopsy not required. N1: Clinically abnormal peripheral lymph nodes; histopathology Dutch grade 1 or National Cancer Institute $(\mathrm{NCl})$ LN0-2. N2: Clinically abnormal peripheral lymph nodes; histopathology Dutch grade 2 or NCI LN3. N3: Clinically abnormal peripheral lymph nodes; histopathology Dutch grades 34 or NCI LN4; clone positive or negative. M1: Visceral involvement (must have pathology confirmation and organ involved should be specified). B0: Absence of significant blood involvement: $5 \%$ or less of peripheral blood lymphocytes are atypical (Sezary cells). B1: Low blood tumor burden: more than $5 \%$ of peripheral blood lymphocytes are atypical (Sezary cells) but does not meet the criteria of B2. B2: High blood tumor burden: 1,000 per microliter or more Sezary cells with positive clone. (AJCC 8th ed.) 